\title{
Van den Ende-Gupta syndrome
}

INSERM

\section{Source}

INSERM. (1999). Orphanet: an online rare disease and orphan drug data base. Van den

Ende-Gupta syndrome. ORPHA:2460

Van den Ende-Gupta syndrome is a very rare syndrome characterized by

blepharophimosis, arachnodactyly, joint contractures, and characteristic dysmorphic features. 